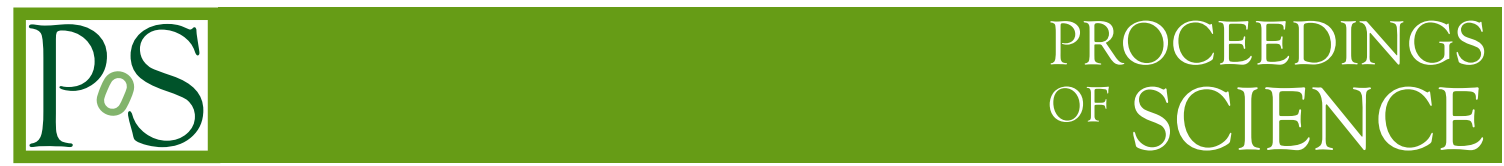

\title{
Development of very slow negative muon beam
}

\author{
Hiroaki Natori* \\ KEK \\ E-mail: hatoriepost.kek.ip
}

Negative muons are utilized as a good probe not only in the elementary particle physics, but also in the non-destructive elemental analysis. Developments of slow negative muon beams are on going in J-PARC to make more sophisticated analysis. Beam tuning down to $2.6 \mathrm{MeV} / \mathrm{c}$ is achieved by detecting slow muons directly with micro-channel plate. Ultra slow negative muons are going to be developed by a novel technique utilizing muon-catalyzed fusion.

The 21st international workshop on neutrinos from accelerators (NuFact2019)

August 26 - August 31, 2019

Daegu, Korea

${ }^{*}$ Speaker. 


\section{Introduction}

Negative muons have been utilized as a good probe in physics. For example, recent measurement of the proton's charge radius with muonic hydrogen [U] drew people's attention as a "proton radius puzzle" until the remeasurement of that for electron's case []. Soon mu-e conversion experiments [3], 团, 回] are expected to be a sensitive probe for the new physics beyond the Standard Model of elementary particle physics. Meanwhile, negative muon is a good probe for a non-destructive analysis of material, too. Its property of having magnetic moment and tendency of emitting electron depending on its spin direction is utilized as a tool of investigation of internal magnetic field of a sample material, called as negative muon spin rotation and relaxation $\left(\mu^{-} \mathrm{SR}\right)$ [可]. In the process of muonic atom formation, characteristic X-rays are emitted which can be utilized for element analysis [Q, [8]. Decay signal of unstable atom after muon capture can be also utilized [Q]. Elemental depth analysis is possible by changing the incident muon momentum. The demand of slow negative muon is increasing to get the information of the surface of the sample to be compared with the other depth. Accelerating very slow muons may be able to offer more precise depth dependence analysis.

\section{Slow muon extraction in D-line of J-PARC}

Figure $\mathbb{W}$ shows a layout of J-PARC muon facility. The Decay/Surface muon beamline, D-line, extracts positive and negative muon beam with a momentum range from several to $120 \mathrm{MeV} / \mathrm{c}$. The D-line has two experimental area. The D1 area is equipped with a set of apparatus to perform $\mu \mathrm{SR}$ analysis, where the users bring their samples for their analysis. And the D2 area is a general purpose area where users bring their equipment to construct their experiments.

In the D-line, warm bore pion capture magnets are adopted and no heat shield window is placed after that. This enables us to extract low momentum decay muons without loss of their energy. In 2019, we updated the low momentum muon extraction in D2 area down to $2.6 \mathrm{MeV} / \mathrm{c}$ from 3.5 $\mathrm{MeV} / \mathrm{c}$ which was achieved in 2016. We improved the efficiency of muon yield measurement by placing a micro-channel plate (MCP) in a vacuum chamber which we connected directly to the beamline, as shown in the Figure \, so that the decay muon beam arrives at the detector without passing through material such as a beam window. In 2016, we placed a muon stopping target and measured characteristic X-ray to estimate the number of coming muons, so the solid angle for the $\mathrm{X}$-ray detector limited the efficiency of muon yield estimation, so the time for the beam tuning got too long to go down to lower momentum. In 2019 experiment, the prompt electrons were clearly identified by the difference of arrival time to the MCP in the estimation of the muon yield. Optimization of currents applied to the quadrupole magnets was done to maximize the muon yield.

Especially in the low momentum case, magnets are operated with much lower current than the usual operation and the residual magnetic fields worsen the reproducibility of the extracted muon yield. Further development of effective operational procedure for the stable reproducibility dedicated to low magnetic current operation's case is on going to cope with the hysteresis of the magnets. 


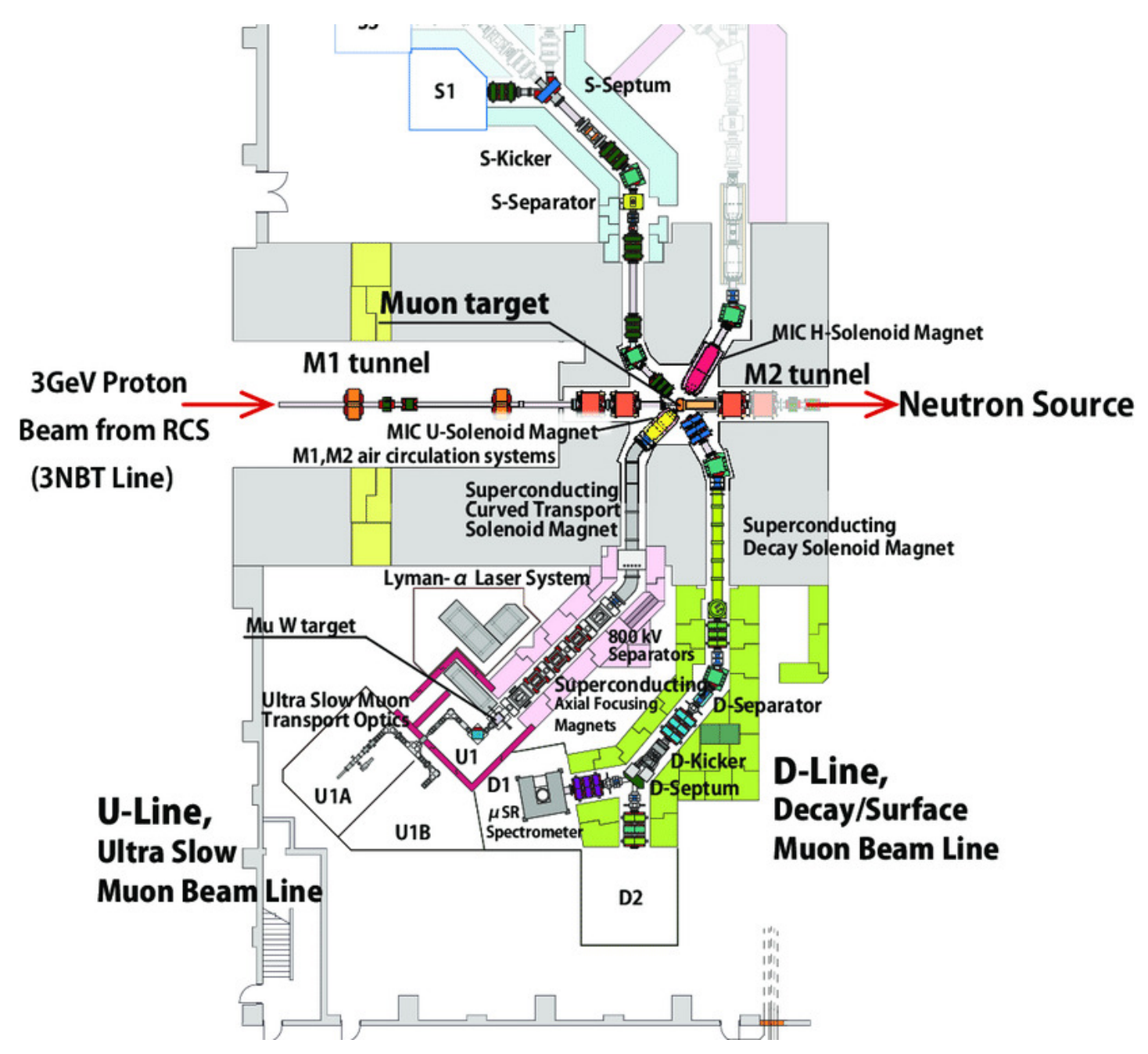

Figure 1: Layout of J-PARC muon beamlines

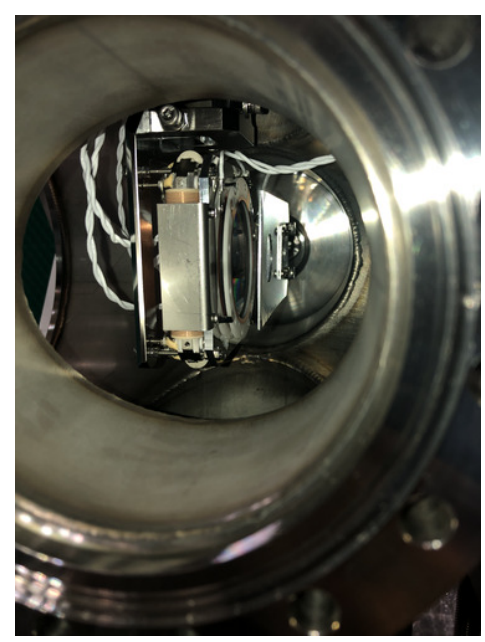

Figure 2: A picture of MCP placed in a vacuum chamber. 


\section{Development of ultra-slow negative muon beam}

The yield of muons gets smaller for the lower momentum. The number of muon extracted to D2 area was estimated to be several per second for the momentum of $2.6 \mathrm{MeV} / \mathrm{c}$. To obtain sufficient muon yield for the lower momentum, we need to investigate a new procedure to generate them.

In the positive muon's case, very slow muon can be obtained by a procedure of stopping muon in a special target material which emits thermal muonium. Then ionizing the muonium with lazer beam and very slow positive muon can be obtained [ए], [1]. But this scheme is difficult for the negative muon because the binding energy of muonic atom is high and muon capture reduces its lifetime.

We are planning a development of a novel way of generating very slow negative muon utilizing muon catalyzed fusion $(\mu \mathrm{CF})$ inspired by a proposal given by K. Nagamine [[2]. When negative muons stop in a condensed $\mathrm{D}_{2} / \mathrm{T}_{2}$ mixture, $\mathrm{dt} \mu$ molecules are made. Then after the reactions of $\mathrm{dt} \mu^{-} \rightarrow \mu^{-5} \mathrm{He} \rightarrow \alpha+\mathrm{n}+\mu^{-}$, free muons with energy around $10 \mathrm{keV}$ are left. As the range of $\sim 10 \mathrm{keV}$ muon is $\sim 3 \mu \mathrm{m}$, only muons which stop very close to the surface can be extracted to be utilized for the elemental analysis, so we need one more procedure to enhance the number of muons to come the surface. To transport muons to the surface, we are planning to utilize RamsauerTownsend effect. The cross section for muonic deuterium $(\mathrm{d} \mu)$ and muonic tritium $(\mathrm{t} \mu)$ to the condensed hydrogen is known to become very small when the energy of $\mathrm{d} \mu$ or $\mathrm{t} \mu$ is a few $\mathrm{eV}$. So we plan to construct muon stopping target with hydrogen with small fraction of $\mathrm{D}_{2}$ or $\mathrm{T}_{2}$ ingredients and cover it with a thin layer of the mixture of $\mathrm{D}_{2} / \mathrm{T}_{2}$. The stopped muons inside the condensed hydrogen forms muonic hydrogen $(\mathrm{p} \mu)$, but $\mathrm{d} \mu$ or $\mathrm{t} \mu$ is more stable, so some fraction of the muons will be transferred to deuterium or tritium $(\mathrm{p} \mu+\mathrm{d} \rightarrow \mathrm{d} \mu+\mathrm{p}, \mathrm{p} \mu+\mathrm{t} \rightarrow \mathrm{t} \mu+\mathrm{p}$ ). The $\mathrm{d} \mu$ or $\mathrm{t} \mu$ have initial energy of $\sim 45 \mathrm{eV}$ and decelerated until it reaches around Ramsauer-Townsend minimum by scattering, then diffuse in the hydrogen with a range of $\sim 0.5 \mathrm{~mm}$. Some of the muons will reach to the thin $\mathrm{D}_{2} / \mathrm{T}_{2}$ layer and extracted to the vacuum after the $\mu \mathrm{CF}$ reactions. P. Strasser et al. performed feasibility study of this scheme using dd reaction [14]] .

The muons extracted to the vacuum have energy around $10 \mathrm{keV}$, and can be collected with high electrical field of $<100 \mathrm{kV}$. Then we plan to perform frictional cooling [[13] to reduce the energy spread. We plan the obtained muons to be utilized for scanning muon microscope using technique similar to that for electron microscope. We expect the beam size to be a few $\mathrm{mm}$ in the stage of collection by the $<100 \mathrm{kV}$ electrical field, then we aim to reduce the beam size to be an order of $10 \mu \mathrm{m}$ by a set of beam optics. We are now preparing experiments to confirm this proposal using tritium which requires approval of treating unsealed source. The experiment should be carefully designed not to leak tritium. We are making design of the experiment taking both the radiation safety and the efficiency of slow muon extraction efficiency into account.

\section{Acknowledgement}

This work is supported by a Grant-in-Aid for Scientific Research on Toward new frontiers "Encounter and synergy of state-of-the-art astronomical detectors and exotic quantum beams" (No. 6007) of the Ministry of Education, Culture, Sports, Science, and Technology, Japan. 


\section{References}

[1] R. Pohl, et al., Nature 466, 213-216 (2010) DOI: 10.1038/nature09250.

[2] N. Bezginov, et al., Science 365, 1007-1012 (2019). DOI: 10.1126/science.aau7807.

[3] H. Natori, DeeMe Collaboration, Nucl. Phys. B Proc. Suppl. 248-250, 52-57 (2014). DOI: 10.1016/j.nuclphysbps.2014.02.010.

[4] COMET Collaboration, arXiv:1812.09018 [physics.ins-det] (2018).

[5] L. Bartoszek, et al., (Mu2e Collaboration), arXiv:1501.05241 [physics.ins-det](2015).

[6] J. Sugiyama, et al., Phys. Rev. Lett. 121, 087202 (2018).

[7] M. Tampo, et al., JPS Conf. Proc. 8, 036016 (2015) DOI:10.7566/JPSCP.8.036016.

[8] K. Ninomiya, et al., Anal. Chem. 2015, 87, 9, 4597-4600 (2015) DOI: 10.1021/acs.analchem.5b01169.

[9] T. Kudo, et al., J. Radioanal. Nucl. Chem. (2019). DOI: 10.1007/s10967-019-06682-8.

[10] K. Nagamine, et al., Phys. Rev. Lett. 74, 4811 (1995).

[11] T. Adachi, et al., PoS (NUFACT2014) 097 (2015).

[12] K. Nagamine, Proc. Japan Academy. Vol. 65, Ser. B (1989) 225.

[13] M. Mühlbauer, et al., Hyp. Interact. 119 (1999), 305.

[14] P. Strasser, et al, Phys. Lett. B 368 (1996) 32-38. 